

E-ISSN: 2706-9575

P-ISSN: 2706-9567

IJARM 2021; 3(2): 224-232

Received: 03-05-2021

Accepted: 13-06-2021

Dr. Sireesha Gunnam Assistant Professor, Department of Nephrology, NRI Medical College/General Hospital, Chinakakani, Guntur, Andhra Pradesh, India

Dr. Shiva Nagendra Reddy Annapareddy

Associate Professor, Department of Nephrology, NRI Medical College, Chinakakani, Guntur, Andhra Pradesh, India
Corresponding Author: Dr. Shiva Nagendra Reddy Annapareddy Associate Professor, Department of Nephrology, NRI Medical College, Chinakakani, Guntur, Andhra Pradesh, India

\section{Spectrum of non-diabetic renal disease in patients with diabetes mellitus in a tertiary care hospital}

\author{
Dr. Sireesha Gunnam and Dr. Shiva Nagendra Reddy Annapareddy
}

DOI: $\underline{\text { https://doi.org/10.22271/27069567.2021.v3.i2d.250 }}$

\begin{abstract}
Aim and Objectives: To analyse frequency and spectrum of non diabetic renal disease (NDRD) in type 2 diabetes patients and also determine the clinical markers associated with NDRD.

Methodology: Single centre based prospective observational study carried out during the period from M a y 2016 to September 2019. Type 2 DM patients with atypical clinical renal disease who underwent renal biopsy to rule out NDRD at King George Hospital, Visakhapatnam were included in the study. Results: In the present study, the overall prevalence of NDRD with or without underlying DN in our study was $71.7 \%$. The prevalence rates of isolated NDRD, NDRD with DN and isolated DN were $51.2 \%, 20.5 \%$, and $28.2 \%$ respectively. Shorter duration of diabetes, absence of retinopathy, presence of active urine sediment are markers associated with NDRD in type 2 diabetics and are strong indicators for biopsy. Isolated DN group patients suffer significantly with hypertension compared to NDRD group. Isolated NDRD group patients had low HbA1c $(<7 \%)$ values compared to isolated DN group. The most common clinical presentations are RPRF, AKI and acute deterioration in renal function. The common lesion found in NDRD patients with or without underlying DN was acute tubulointerstitial nephritis. In Isolated NDRD group, primary glomerular diseases were most common. IgA Nephropathy was the most common glomerular disease in our study.

Conclusion: To conclude, it is difficult to differentiate NDRD from DN merely on the basis of clinical and laboratory criteria and kidney biopsy is an important diagnostic tool to define underlying disease and to identify the treatable causes.
\end{abstract}

Keywords: Diabetic nephropathy, NDRD, AKI

\section{Introduction}

Diabetic nephropathy (DN)/Diabetic kidney disease (DKD) is one of the major complications of diabetes mellitus (DM). Diabetic renal disease develops in about 20-40\% of patients suffering from diabetes mellitus (DM) ${ }^{[1,2]}$ It is the leading cause of end-stage renal disease (ESRD) worldwide ${ }^{[3]}$. The prognosis in such patients is poor compared with patients with ESRD due to other renal diseases ${ }^{[4,5]}$. Unlike type $1 \mathrm{DM}$ patients where $>95 \%$ of renal disease is the result of diabetic nephropathy (DN), the renal disease in type 2DM (T2DM) patients is more complex and heterogeneous. Kidney diseases other than diabetic nephropathy in type 2 diabetic patients are known as non-diabetic renal disease (NDRD), either isolated or superimposed on DN ${ }^{[6]}$. The occurrence of NDRD in diabetic patients is reported in several studies from around the world ${ }^{[7-10]}$. Although exact incidence of NDRD is not known, frequency varies from $5 \%$ to $71 \%$ in various studies ${ }^{[11]}$. It is seen in $26.7 \%$ of Asian and $22 \%$ of European patients $[9,12,13]$. A wide spectrum of NDRD, including both glomerular and tubulointerstitial lesions are reported, the precise diagnoses of which require renal biopsy. The clinical markers indicating the presence of NDRD include abrupt onset of overt proteinuria, rapid decline in renal function, impaired renal function without significant proteinuria, absence of neuropathy, absence of retinopathy and presence of active urinary sediment, or features of other systemic diseases ${ }^{[14,15]}$. DN is hard to reverse. However, certain NDRD, are often treatable, even remittable ${ }^{[8,9]}$. Since diabetic patients with NDRD have significantly better renal outcomes compared to patients with biopsy proven DN, it is important to identify, and manage NDRD as early as possible ${ }^{[16]}$. Hence this study was done to determine the spectrum of NDRD in type 2 diabetes patients. 


\section{Aims and Objectives}

1. To analyze frequency and spectrum of non diabetic renal disease(NDRD) in type 2 diabetes patients with atypical clinical features

2. To determine the clinical markers associated with NDRD.

\section{Materials and Methods \\ Study Design: Single centre based prospective observational study.}

Study period: May 2016 to September 2019.

\section{Inclusion criteria}

Type 2 DM patients with atypical clinical renal disease (abrupt onset of proteinuria, active urinary sediment, rapid deterioration in renal function, renal dysfunction without significant proteinuria, features of other systemic diseases, Nephrotic syndrome with normal function, RPRF), who underwent renal biopsy to rule out NDRD at King George Hospital, Visakhapatnam were included in the study

\section{Exclusion criteria}

End stage renal disease on renal replacement therapy has been excluded. Pregnancy excluded.

Patients with inadequate renal biopsies were excluded.

\section{Methods}

The Demographic, clinical and biochemical data collected from these patients. Thorough history was taken and physical examination done for these patients. The diagnosis of diabetes mellitus was made according to the criteria stated by the American Diabetes Association.

Clinical details and laboratory parameters including age, age at onset (the time when DM was first diagnosed), duration of diabetes (the period between the age of onset and renal biopsy), blood urea, serum creatinine, Serum albumin, complete urine examination, $24 \mathrm{hr}$ urine protein, HbA1c, lipid profile, presence or absence of retinopathy, presence or absence of neuropathy, presence or absence of hypertension were recorded at the time of renal biopsy. Chest x-ray, ECG, USG Abdomen, 2D Echo were done routinely. Special investigations like collagen profile, serum complement levels, ASO titres, C-reactive protein were done based on clinical circumstances.

Renal biopsy was performed under ultrasound guidance with a biopsy gun (BARD gun 16/18 gauge, 22mm cutting edge). Examination of renal biopsy tissue was done by light microscopy (hematoxylin and eosin ( $\mathrm{H}$ and $\mathrm{E})$, periodic acid Schiff (PAS), and methenamine silver staining) and immunofluorescence microscopy using fluorescein isothiocyanate (FITC) conjugated rabbit anti-human immunoglobulin (Ig) G, IgM, IgA, and C3 antibodies. The intensity was semi quantitatively scored as 0 for negative, $1+$ for present, 2+ for definite, 3+ for strongly positive. Electron microscopy was not used as this facility is not available to us. DN was diagnosed when at least three of the following features were present; (i) global mesangial sclerosis with or without Kimmelstiel- Wilson nodule or nodular mesangial sclerosis, (ii) uniform glomerular capillary basement membrane thickening, (iii) exudative lesions such as "fibrin cap" or "capsular drop", or (iv) glomerular hyaline arteriolosclerosis [17]. Based on the biopsy and direct immunofluorescence findings, patients were grouped as group 1, isolated NDRD, group 2, NDRD with underlying DN(Mixed lesions) and group 3, isolated DN.

Statistical analysis: Descriptive statistical methods were used. For metric data, arithmetic mean, standard deviation, and median were used. For categorical data, Fisher's exact test was applied. Student T- test was used for continuous variables. A Differences of various parameters between the three groups were assessed by analysis of variance (ANOVA) test and Chi-square test of statistical significance. A P value $<0.05$ was considered statistically significant. Statistical analysis was done by statistical software SPSS for Windows v17.0 (SPSS, Chicago, IL, USA).

\section{Results}

A total of 40 patients were identified, of which 1 was excluded as the patient had advanced renal failure at the time of renal biopsy which showed end stage nephrosclerosis, the primary cause of which could not be clearly delineated as DN or NDRD. The remaining 39 patients were included in the study. They are divided in to 3 groups. Group 1 include patients with isolated NDRD, Group 2 patients had NDRD with underlying DN (Mixed lesions), Group 3 patients had isolated DN.

Out of these 39 patients, twenty patients (51.2\%) had isolated NDRD (Group 1), eight patients (20.5\%) had NDRD with underlying DN (Group 2), eleven patients (28.2\%) had isolated DN (Group 3)

Table 1: Distribution of cases in groups

\begin{tabular}{|c|c|c|}
\hline Group & Frequency & Percent \\
\hline Isolated NDRD (Group1) & 20 & 51.2 \\
\hline NDRD + DN (Group 2) & 8 & 20.5 \\
\hline Isolated DN (Group 3) & 11 & 28.2 \\
\hline Total & 39 & 100.0 \\
\hline
\end{tabular}

The mean age \pm SD among these patients is $46.43 \pm 10.3$ years. Out of them, 24 were females $(61.5 \%), 15$ were males (38.5\%).Male to female ratio was 1:1.6.

Table 2: Gender distribution among patients

\begin{tabular}{|c|c|c|c|}
\hline & Frequency & Percent & P Value \\
\hline Male & 15 & 38.5 & \\
\cline { 1 - 3 } Female & 24 & 61.5 & \multirow{2}{*}{$>0.05$} \\
\hline Total & 39 & 100.0 & \\
\hline
\end{tabular}

Females are more in number than males which is statistically not significant. The mean age among females is $46 \pm 12$ years and among males is $48 \pm 8$ years. There is no significant mean age difference between Males and Females. Among group 1 patients, males were 7(46.7\%), females were $13(54.2 \%)$ and male to female ratio was $1: 1.8$. Among group 2 patients, males were 2(13.3\%), females were $6(25 \%)$ and male to female ratio was 1:3. Among group 3 patients, males were $6(40 \%)$, females were $5(20.8 \%)$ and male to female was $1.2: 1$. 
Table 3: Gender distribution in each group

\begin{tabular}{|c|c|c|c|c|c|c|}
\hline \multicolumn{2}{|c|}{} & Isolated NDRD (Group 1) & NDRD + DN (Group 2) & Isolated DN (Group 3) & Total & P Value \\
\hline \multirow{2}{*}{ Females } & No. of patients & 13 & 6 & 5 & 24 \\
\cline { 2 - 6 } & Percentage & $54.2 \%$ & $25.0 \%$ & $20.8 \%$ & $100.0 \%$ \\
\hline \multirow{2}{*}{ Males } & No. of patients & 7 & 2 & 6 & 15 \\
\cline { 2 - 7 } & Percentage & $46.7 \%$ & $13.3 \%$ & $40.0 \%$ & $100.0 \%$ \\
\hline Total & Count & 20 & 8 & 11 & 39 & \\
\hline
\end{tabular}

Females are more in isolated NDRD (Group 1) and NDRD+DN group (Group 2) whereas Males are more in isolated DN group (Group 3) even though it is not statistically significant.

The following table 12 depicts the clinical and biochemical parameters of the three groups of patients. There was no statistically significant difference in age of patients, $\mathrm{S}$. creatinine, $24 \mathrm{~h}$ urine protein $(P>0.05)$. Statistically significant difference was present among the groups for the duration of diabetes and serum albumin. Patients with isolated NDRD (Group 1) had shorter duration of diabetes compared to the other groups $(P=0.046)$.Patients with isolated DN (Group 3) had low serum albumin compared to other groups $(\mathrm{P}=0.043)$.

Table 4: Biochemical and clinical parameters among different groups

\begin{tabular}{|c|c|c|c|c|}
\hline & Isolated NDRD (Group 1) & NDRD + DN (Group 2) & Isolated DN (Group 3) & \multirow{2}{*}{ P Value } \\
\cline { 2 - 5 } & Mean \pm SD & Mean \pm SD & Mean \pm SD & $.73 .00 \pm 5.18$ \\
\hline Age & $46.75 \pm 11.92$ & $48.88 \pm 11.91$ & $8.73 \pm 4.94$ & $.046 *$ \\
\hline Duration Of DM & $4.60 \pm 4.45$ & $5.13 \pm 2.85$ & $5.22 \pm 3.54$ & .806 \\
\hline Serum Creatinine & $4.98 \pm 2.91$ & $5.80 \pm 2.12$ & $2.63 \pm 0.63$ & $.043^{*}$ \\
\hline Serum Albumin & $3.21 \pm 0.71$ & $2.70 \pm 0.56$ & $2.12 \pm 1.57$ & .713 \\
\hline 24HrUrineProtein & $1.56 \pm 0.77$ & $1.56 \pm 0.77$ & & \\
\hline
\end{tabular}

Among 39 patients, 27 patients (69.2\%) had hypertension. Among group 1 patients $10(50 \%)$ had hypertension, in group 2 patients $7(87.5 \%)$ had hypertension, in group 3 patients 10(90.9\%) had hypertension. NDRD+DN and Isolated DN groups patients suffer significantly from HTN when compared to the other which is statistically significant
$(P<0.05)$. In our study, isolated NDRD group (Group 1) patients had high number of patients with low HbA1c $(<7 \%)$ when compared to patients with isolated DN (Group $3)$ who had high HbA1c values $(>7 \%)$ which is statistically significant $(P<0.05)$.

Table 5: Hypertension among different groups

\begin{tabular}{|c|c|c|c|c|c|}
\hline & & \multicolumn{3}{|c|}{ HTN } & \multirow{2}{*}{ P Value } \\
\hline & & Present & Absent & Total & \\
\hline \multirow{2}{*}{ Isolated NDRD (Group 1) } & No. of patients & 10 & 10 & 20 & \multirow{8}{*}{$<0.05$} \\
\hline & Percentage & $50.0 \%$ & $50.0 \%$ & $100.0 \%$ & \\
\hline \multirow{2}{*}{ NDRD + DN (Group 2) } & No. of patients & 7 & 1 & 8 & \\
\hline & Percentage & $87.5 \%$ & $12.5 \%$ & $100.0 \%$ & \\
\hline \multirow{2}{*}{ Isolated DN (Group 3) } & No. of patients & 10 & 1 & 11 & \\
\hline & Percentage & $90.9 \%$ & $9.1 \%$ & $100.0 \%$ & \\
\hline \multirow{2}{*}{ Total } & No. of patients & 27 & 12 & 39 & \\
\hline & Percentage & $69.2 \%$ & $30.8 \%$ & $100.0 \%$ & \\
\hline
\end{tabular}

Table 6: Hba1c values among different groups

\begin{tabular}{|c|c|c|c|c|c|c|c|c|}
\hline & \multicolumn{2}{|c|}{ Isolated NDRD (Group 1) } & \multicolumn{2}{|c|}{ NDRD + DN (Group 2) } & \multicolumn{2}{|c|}{ Isolated DN (Group 3) } & \multirow{2}{*}{ P Value } \\
\hline & & No. of patients & Percentage & No. of patients & Percentage & No. of patients & Percentage & \\
\hline \multirow{2}{*}{ HbAlc } & $<7$ & 15 & $38.5 \%$ & 0 & $0.0 \%$ & 1 & $2.6 \%$ & \multirow{2}{*}{$<0.05 \mathrm{Sig}$} \\
\hline & $>7$ & 5 & $12.8 \%$ & 8 & $20.5 \%$ & 10 & $25.6 \%$ & \\
\hline
\end{tabular}

Indications for renal biopsy include: acute deterioration in renal function in 8 patients $(20.5 \%)$, RPRF (Rapidly Progressive Renal Failure) in 8 patients (20.5\%), AKI (Acute Kidney Injury) in 8 patients (20.5\%), Nephrotic syndrome in 6 patients (15.4\%), Active urine sediment in 4 patients $(10.3 \%)$, Abrupt onset of proteinuria in 3 patients (7.7\%), Renal failure without significant proteinuria in 2 patients $(5.1 \%)$.

Table 7: Various clinical presentations

\begin{tabular}{|c|c|c|}
\hline Clinical Presentation & Frequency & Percent \\
\hline Acute deterioration in renal function & 8 & 20.5 \\
\hline AKI & 8 & 20.5 \\
\hline RPRF & 8 & 20.5 \\
\hline Active Urine Sediment & 4 & 10.3 \\
\hline Nephrotic syndrome & 6 & 15.4 \\
\hline Abrupt onset of proteinuria & 3 & 7.7 \\
\hline Renal failure without significant proteinuria & 2 & 5.1 \\
\hline Total & 39 & 100.0 \\
\hline
\end{tabular}


Table 8: Clinical presentation among different groups

\begin{tabular}{|c|c|c|c|c|}
\hline Clinical Presentation & Isolated NDRD (Group 1) & NDRD + DN (Group 2) & DN (Group 3) & Total \\
\hline Acute deterioration in renal function & 3 & 2 & 3 & 8 \\
\hline AKI & 6 & 2 & 0 & 8 \\
\hline RPRF & 4 & 4 & 0 & 8 \\
\hline Nephrotic syndrome & 3 & 0 & 3 & 6 \\
\hline Active urine sediment & 3 & 0 & 1 & 4 \\
\hline Abrupt onset of proteinuria & 0 & 0 & 3 & 3 \\
\hline Renal failure without significant proteinuria & 1 & 0 & 1 & 2 \\
\hline Total & 20 & 8 & 11 & 39 \\
\hline
\end{tabular}

Retinopathy was present in 12 cases. 2 patients of group 1, 6 patients of group 2 and 4 patients of group 3 had retinopathy at the time of renal biopsy. Presence of Retinopathy is highest in NDRD+DN and isolated DN groups when compared to the other which is statistically significant $(P<$ $0.05)$. Presence of Active sediment is highest in NDRD and NDRD + DN groups when compared to the other which is statistically significant.

Table 9: Retinopathy among different groups

\begin{tabular}{|c|c|c|c|c|c|c|c|}
\hline & & & Isolated NDRD (Group 1) & NDRD + DN (Group 2) & Isolated DN (Group 3) & Total & \\
\hline \multirow{4}{*}{ Retinopathy } & & No. of patients & 2 & 6 & 4 & 12 & $P$ value \\
\hline & Present & Percentage & $16.7 \%$ & $50.0 \%$ & $33.3 \%$ & $100.0 \%$ & \multirow{5}{*}{$<0.05$} \\
\hline & & No. of patients & 18 & 2 & 7 & 27 & \\
\hline & AUSCAIt & Percentage & $66.7 \%$ & $7.4 \%$ & $25.9 \%$ & $100.0 \%$ & \\
\hline \multirow{2}{*}{\multicolumn{2}{|c|}{ Total }} & No. of patients & 20 & 20 & 11 & 39 & \\
\hline & & Percentage & $51.3 \%$ & $51.3 \%$ & $28.2 \%$ & $100.0 \%$ & \\
\hline
\end{tabular}

Table 10: Urine active sediment in each group

\begin{tabular}{|c|c|c|c|c|c|c|c|}
\hline & & & Isolated NDRD (Group 1) & NDRD + DN (Group 2) & Isolated DN (Group 3) & \begin{tabular}{|l|} 
Total \\
\end{tabular} & $\mathbf{P}$ \\
\hline \multirow{4}{*}{$\begin{array}{c}\text { Urine Active } \\
\text { sediment (UAS) }\end{array}$} & Precent & No. of patients & 7 & 5 & 1 & 13 & Value \\
\hline & Present & Percentage & $53.8 \%$ & $38.5 \%$ & $7.7 \%$ & $100.0 \%$ & \multirow{5}{*}{$<0.05$} \\
\hline & \multirow{2}{*}{ Absent } & No. of patients & 13 & 3 & 10 & 26 & \\
\hline & & Percentage & $50.0 \%$ & $11.5 \%$ & $38.5 \%$ & $100.0 \%$ & \\
\hline \multirow{2}{*}{\multicolumn{2}{|c|}{ Total }} & No. of patients & 20 & 20 & 11 & 39 & \\
\hline & & Percentage & $51.3 \%$ & $51.3 \%$ & $28.2 \%$ & $100.0 \%$ & \\
\hline
\end{tabular}

Other micro and macro vascular complications include CAD in 4 patients $(10.2 \%)$, peripheral neuropathy in 4 patients (10.2\%), CVA in 1 patient $(2.5 \%)$.

The most common lesion found in NDRD patients with or without concurrent DN was acute tubulointerstitial nephritis (ATIN) in $7(25 \%)$ cases (4 in group 1 and 3 in group 2) followed by IgA Nephropathy in 4(14.2\%) cases.

Group 1 (Isolated NDRD): NDRD was found in 51.2\% (20 Of 39 patients) of patients. Among them 45\% (9) had primary glomerular diseases (PGD), 30\% had tubulointerstitial diseases (TID), $15 \%$ (3) had secondary glomerular diseases (SGD), 10\% (2) had vascular disease. IgA Nephropathy was the most common PGD affecting $33.3 \%$ (3) of participants, followed by FSGS (Focal and Segmental Glomerulosclerosis) in $22.2 \%(2)$, IRGN (Infection related Glomerulonephritis) in $11.1 \%(1)$, MCD (Minimal Change Disease) in $11.1 \%(1)$, Membranous nephropathy in $11.1 \%(1)$, MPGN in $11.1 \%$ (1) of patients. ATIN was the most common TID found in $66.6 \%$ (4) of patients, followed by ATN in $16.6 \%$ (1), Cast nephropathy in $16.6 \%$ (1) patients. The secondary glomerular diseases are lupus nephritis affecting $33.3 \%$ (1), antineutrophil cytoplasmic antibody (ANCA) related pauci-immune glomerulonephritis in $33.3 \%$ (1), TMA (Thrombotic Microangiopathy) in $33.3 \%$ (1) of patients. In vascular disease, benign nephrosclerosis was present in $20 \%$ of patient

Group 2 (NDRD + DN): NDRD with underlying DN was found in $20.5 \%$ ( 8 of 39 patients) of patients. Among them $62.5 \%$ (5) had tubulointerstitial diseases (TID), 37.5\% (3) had primary glomerular diseases (PGD). ATIN was the most common TID found in $60 \%$ (3) of patients, followed by acute tubular necrosis in $40 \%$ (2). IRGN was the most common PGD found in $66.6 \%$ (2), followed by IgA Nephropathy in $33.3 \%$ (1) of patients.

Group 3 (Isolated DN): Though clinically suspected to have NDRD, 28.2\% (11 of 39 participants) were diagnosed to have isolated DN. Among them, 72.7\% (8) had Kimmelsteil Wilson nodules (DN -RPS class 3), 18.1\% (2) has DN-RPS class 4 lesions, 9\% (1) had DN- RPS class $2 b$ lesions.

Table 11: Spectrum of NDRD in type 2 diabetic patients

\begin{tabular}{|c|c|c|c|c|}
\hline \multirow{2}{*}{ Renal Disease } & & $\begin{array}{c}\text { NDRD (Group 1) No. } \\
\text { of patients }\end{array}$ & $\begin{array}{c}\text { NDRD + DN (Group 2) } \\
\text { No. of patients }\end{array}$ & Total percentage \\
\hline Primary Glomerular Diseases & IgA Nephropathy & 3 & 1 & $14.2 \%(4)$ \\
\hline & IRGN & 1 & 2 & $10.7 \%(3)$ \\
\cline { 2 - 5 } & FSGS & 2 & 0 & $7.1 \%(2)$ \\
\cline { 2 - 5 } & Minimal Change disease & 1 & 0 & $3.5 \%(1)$ \\
\hline
\end{tabular}




\begin{tabular}{|c|c|c|c|c|}
\hline & Membranous Nephropathy & 1 & 0 & $3.5 \%(1)$ \\
\cline { 2 - 5 } & MPGN & 1 & 0 & $3.5 \%(1)$ \\
\hline Secondary Glomerular Diseases & Lupus Nephritis & 1 & 0 & $3.5 \%(1)$ \\
\hline & ANCA Vasculitis & 1 & 0 & $3.5 \%(1)$ \\
\cline { 2 - 5 } & TMA & 1 & 0 & $3.5 \%(1)$ \\
\hline Tubulointerstitial diseases & Acute tubulointerstitial nephritis & 4 & 3 & $25 \%(7)$ \\
\hline & Acute tubular necrosis & 1 & 2 & $10.7 \%(3)$ \\
\cline { 2 - 5 } & Cast nephropathy & 1 & 0 & $3.5 \%(1)$ \\
\hline Vascular Disease & Benign nephrosclerosis & 2 & 0 & $7.1 \%(2)$ \\
\hline Total & & 20 & 8 & $100 \%$ \\
\hline
\end{tabular}

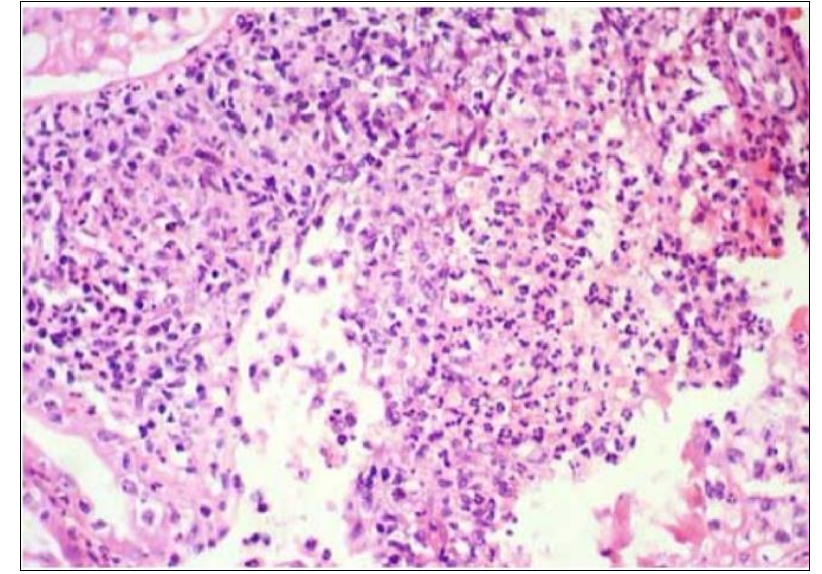

Fig 1: Acute tubulointerstitial nephritis (H \& E, X400) (Patient no: 3 in excel sheet)

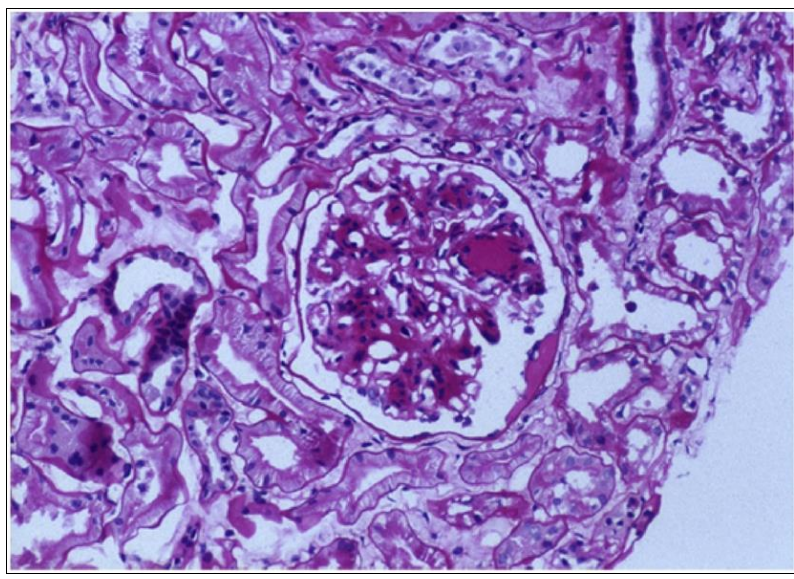

Fig 2: The glomerulus shows mesangial expansion, nodule formation, prominent basement membranes and capsular drop, all indicative of diabetic nephropathy (PAS, X100) (Patient no: 9 in excel sheet)

\section{Discussion}

Diabetic Nephropathy is one of the most frequent and clinically significant complications of diabetes mellitus. It affects approximately $40 \%$ of patients who have had diabetes for $>20$ years and has become a major cause of ESRD worldwide ${ }^{[1,3]}$. Although patients with type $2 \mathrm{DM}$ (T2DM) often experience DN, they can also develop other renal diseases, pathologically unrelated to diabetes known as NDRD ${ }^{[6,8]}$. A wide spectrum of NDRD, including both glomerular and tubule-interstitial lesions are reported in patients with T2DM ${ }^{[7-9]}$. Interestingly, among patients with type 2 diabetes who have undergone renal biopsy, the prevalence of NDRD varies widely from $12 \%$ to $81 \%$, depending on the selection criteria and the population being studied ${ }^{[18]}$.

In this study, 40 patients were identified, of which 1 was excluded as the patient had advanced renal failure at the time of renal biopsy which showed end stage nephrosclerosis, the primary cause of which could not be clearly delineated as DN or NDRD. The remaining 39 patients were included in the study. In the present study, the prevalence rates of isolated NDRD (Group 1), NDRD with underlying DN (Group 2) and isolated DN (Group 3) were $51.2 \%, 20.5 \%$, and $28.2 \%$ respectively. So, the overall prevalence of NDRD with or without underlying DN in our study was $71.7 \%$.

This was in accordance with previous studies conducted by Soni et al., ${ }^{[7]}$ south India (72.5\%); Pham et al., ${ }^{[10]}$ USA (72.5\%); Chang et al., ${ }^{[9]}$ Korea (63.9\%); but different from other studies where the prevalence of NDRD was around $12.3-33.3 \%{ }^{[2]}$. The prevalence rates of NDRD, DN, and NDRD + DN varied from $24.73 \%$ to $82.9 \%, 6.5 \%$ to $66 \%$, and $4 \%$ to $44.08 \%$, respectively, in earlier studies ${ }^{[20,21]}$.

The large variations in percentages are due to different indications for doing renal biopsy, geographical and ethnic factors in these patients ${ }^{[8]}$. The real prevalence of NDRD is difficult to define because renal biopsy is generally performed when clinical presentation is atypical. Consequently, NDRD prevalence in T2DM is often overestimated.

Table 12: Comparison of prevalence of NDRD in different studies

\begin{tabular}{|l|c|c|c|c|}
\hline & Present study & $\begin{array}{c}\text { Soni } \text { et al.. }{ }^{[7]} \\
\text { (South India) }\end{array}$ & $\begin{array}{c}\text { Pham } \text { et al. } .{ }^{[10]} \\
\text { (USA) }\end{array}$ & $\begin{array}{c}\text { Li } \text { et al.. }{ }^{[24]} \\
\text { (China) }\end{array}$ \\
\hline Prevalence & $71.7 \%$ & $72.5 \%$ & $72.5 \%$ & $75.5 \%$ \\
\hline
\end{tabular}

In the present study, the mean age \pm SD among these patients is $46.43 \pm 10.3$ years. The mean age among females is $46 \pm 12$ years and among males is $48 \pm 8$ years. There is no significant mean age difference between Males and Females. Unnikrishnan et al., covering south Indian population reported the average age of patients as $51 \pm 12$ years ${ }^{[22]}$. Mak et al., ${ }^{[8]}$ reported average age was $57 \pm 1.8$ years in patients having DN and $50 \pm 1.9$ years in patients having mixed lesions in their study.

In the present study, $61.5 \%$ were females (24), $38.5 \%$ were males (15). Male to female ratio was $1: 1.6$. Unlike certain studies ${ }^{[1,8]}$. Where gender was comparable between NDRD and DN groups, in the present study female gender was more common in diabetics with isolated NDRD (Group 1) and NDRD with underlying DN (Group 2) groups rather than diabetics with isolated DN (Group 3), even though it is not statistically significant. The male to female ratio in isolated NDRD, NDRD +DN and isolated DN groups were $1: 1.8,1: 3$ and 1.2:1 respectively. So, in our study females were more common in isolated NDRD group than isolated DN group. This was comparable with previous studies done by Soni et al., ${ }^{[7]}$ and Chang et al., ${ }^{[16]}$ In a prospective observational study done by Gall et al., ${ }^{[29]}$ involving 176 patients with type 2 diabetes reported that males had a 2.6 
times greater risk of developing incipient or overt nephropathy.

In this study, we found that patients with isolated NDRD (Group 1) had shorter duration of diabetes (4.6 $\pm 4.4 \mathrm{yrs})$ compared to isolated DN group (Group 3) (8.7 \pm 4.9 yrs) with statistically significant difference $(P=0.046)$. The mean duration of diabetes in patients with NDRD with underlying $\mathrm{DN}$ (Group 2) is $5.13 \pm 2.8 \mathrm{yrs}$. This finding was similar to study done by Chang et al. ${ }^{[16]}$ reported a mean diabetes duration of 5.9 years in patients with NDRD versus 10.6 years in patients with DN alone ( $P<0.001$ for comparison). In study done by K. Kritmetapak et al., ${ }^{[23]}$ found that the mean duration of diabetes was significantly longer in patients with isolated $\mathrm{DN}(8.16 \pm 4.5$ years $)$ than in patients with NDRD with or without DN (5.36 \pm 2.9 years). So, short duration of diabetes ( $<8$ years) had a high likelihood of predicting NDRD versus isolated DN.

Table 13: Comparison of mean duration of diabetes among different studies

\begin{tabular}{|c|c|c|c|c|c|c|}
\hline & \multicolumn{2}{|c|}{ Present study } & \multicolumn{2}{|c|}{ Chang et al., ${ }^{[16]}$} & \multicolumn{2}{|c|}{ K Kritmetapak et al. ${ }^{[23]}$} \\
\hline & NDRD group & DN group & NDRD group & DN group & NDRD group & DN group \\
\hline Mean duration of diabetes (Yrs) & $4.6 \pm 4.4$ & $8.7 \pm 4.9$ & 5.9 & 10.6 & $5.3 \pm 2.9$ & $8.1 \pm 4.5$ \\
\hline
\end{tabular}

Proteinuria is an independent predictor for adverse renal outcome not only in type $2 \mathrm{DM}$ patients, but also in patients with superimposed or isolated NDRD ${ }^{[17]}$. In present study, average $24 \mathrm{hr}$ urine protein was slightly higher in isolated DN group $(2.12 \pm 1.57 \mathrm{gm})$ when compared to isolated NDRD group $(1.56 \pm 0.7 \mathrm{gm})$. But this difference was not statistically significant. So, we found comparable levels of $24 \mathrm{~h}$ protein among the different groups with no statistically significant difference.

This was in accordance with results of $\mathrm{Li}$ et al., ${ }^{[24]}$ and Castellano et al., [25] whereas other studies [26] reported significantly lower levels of proteinuria in NDRD cases as compared with diabetics with DN. So, analysis of proteinuria differs from one study to another.

In the present study, there was no statistically significant difference in age of patients, S. creatinine, $24 \mathrm{~h}$ urine protein between three groups $(P>0.05)$. This was similar to Ghani et al., ${ }^{[1]}$ and Mak et al. ${ }^{[8]}$ who showed no statistically significant difference for age, age at onset of diabetes, blood urea nitrogen levels and serum creatinine.

In our study, $69.2 \%$ (27) of the patents had hypertension. Patients with isolated DN suffer significantly from hypertension when compared to isolated NDRD which is statistically significant $(P<0.05)$. This was similar to study done by Zhou et al. ${ }^{[20]}$, who found that higher systolic blood pressure was a predictor factor of DN.

In our study, NDRD group patients had high number of patients with low HbAlc $(<7 \%)$ when compared to patients with isolated DN who had high HbA1c values (>7\%) which is statistically significant $(\mathrm{P}<0.05)$.This is comparable with a recent Chinese study who identified duration of DM, systolic blood pressure, HbA1c, hematuria, and retinopathy as the five major differential indicators ${ }^{[20]}$.

The most common Indication for renal biopsy in our study are RPRF (20.5\%), AKI (20.5\%), acute deterioration in renal function $(20.5 \%)$.Other indications are Nephrotic syndrome in $15.4 \%$, Active urine sediment in $10.3 \%$, Abrupt onset of proteinuria in $7.7 \%$, Renal failure without significant proteinuria in $5.1 \%$ of the patients. This is agreeing with majority of the published studies ${ }^{[7]}$. Contrary to this, Prakash et al. ${ }^{[12]}$ reported CRF as the most common clinical presentation (47\%) followed by Acute Nephritic Syndrome (18.7\%) and Nephrotic Syndrome (15.6\%). A recent study from Taiwan observed AKI (58.8\%) in majority of patients while in Kuwait, it was hematuria ${ }^{[27]}$. From these review of literatures, it is obvious that presenting syndrome can be different. The indications for renal biopsy in type $1 \mathrm{DM}$ has been somewhat established but the indications are not as clear in patients with type 2 DM.

In our study, all the patients who presented with RPRF and AKI had underlying NDRD with or without superimposed DN. In patients presenting with AKI, $75 \%$ had isolated NDRD and $25 \%$ had NDRD with underlying DN. In patients presenting with RPRF, 50\% had isolated NDRD and $50 \%$ had NDRD with underlying DN. Interestingly, all of our patients presenting with abrupt onset of proteinuria actually had isolated DN on renal biopsy. A plausible explanation for this finding is that the majority of our patients had longstanding poorly controlled diabetes with established microvascular complications (e.g. diabetic retinopathy). These patients may develop progressively increasing albuminuria faster than other patients.

Microscopic hematuria was present in $12.8 \%$ (5) of patients. Among them, $60 \%$ had isolated NDRD and $20 \%$ had mixed lesions (NDRD + DN). So, presence of Active sediment is highest in NDRD and NDRD with DN groups when compared to isolated DN which is statistically significant $(P<0.05)$. The strong correlation of the latter with NDRD is presumably related to the occurrence of proliferative glomerulonephritis (IgA nephropathy) in the NDRD group. This is similar to Mak et al. ${ }^{[8]}$ and Lee et al. ${ }^{[9]}$ who observed microscopic hematuria as an important predictor of NDRD which is also supported by a recent study from China ${ }^{[8,9,20]}$. It is worth mentioning here that about $20 \%$ of our patients with isolated DN had active urinary sediment. Okada et al. ${ }^{[28]}$ found an association between the presence of arteriolar hyalinosis in renal biopsy and persistent microscopic hematuria in type 2 diabetes patients. Matsumura et al. ${ }^{[29]}$ reported that diabetic patients with glomerular hematuria exhibited histologically advanced diffuse lesions, nodular lesions, micro aneurysms, crescent formation, capsular adhesion and interstitial lesions more often than those without hematuria.

In our study, diabetic retinopathy was present in $30.7 \%$ of patients. We observed that diabetic retinopathy was absent in $90 \%$ of the patients with isolated NDRD (Group 1) and present in $75 \%$ of patients with mixed lesions(Group 2 NDRD with DN) and present in $36.3 \%$ of patients with isolated DN (Group 3). So, presence of Retinopathy is highest in NDRD+DN and DN groups when compared to isolated NDRD which is statistically significant $(\mathrm{P}<0.05)$. In patients with retinopathy, $16.7 \%$ had isolated NDRD and in patients without retinopathy, $25.9 \%$ had isolated DN. So, the absence of retinopathy favours NDRD but does not exclude occurrence of DN. This result was in accordance with previous studies conducted by Ghani et al., ${ }^{[1]}$. 
DR is present virtually in all type 1 diabetic patients with DN, whereas only $50-60 \%$ of proteinuric type 2 diabetic patients have retinopathy ${ }^{[30]}$. Absence of retinopathy should prompt further investigation for non-diabetic glomerular diseases ${ }^{[31]}$.

Castellano et al., ${ }^{[25]}$ found that retinopathy had a predictive value of $100 \%$ in predicting $\mathrm{DN}$ and concluded that its existence makes renal biopsy procedure to rule out NDRD unnecessary. However, as even $16.7 \%$ of isolated NDRD patients in the current study had retinopathy, its existence does not obviate the need for renal biopsy, especially if the clinical presentation is atypical. Retinopathy strongly correlates with presence of $\mathrm{DN}$ and considered as an important predictor for adverse renal outcome and disease progression ${ }^{[17]}$.

In the present study, isolated DN patients with retinopathy had higher levels of proteinuria and serum creatinine compared to those with absent retinopathy. Other micro and macro vascular complications observed in this study include CAD in 4 patients $(10.2 \%)$, peripheral neuropathy in 4 patients $(10.2 \%)$, CVA in 1 patient $(2.5 \%)$.

The most common NDRDs varied in different studies, due to variations in biopsy policies, geographic and ethnic factors. In the present study, the most common lesion found in NDRD patients with or without underlying DN was acute tubulointerstitial nephritis in 7(25\%) cases (4 in group 1 and 3 in group 2) followed by $\operatorname{IgA}$ Nephropathy in $4(14.2 \%)$ cases (3 in group 1 and 1 in group 2). In present study, 15 cases $(53.5 \%)$ of the NDRD group (with or without underlying DN) had glomerular diseases, 11 cases $(39.2 \%)$ had tubulointerstitial diseases and 2 cases (7.1\%) had vascular disease.

In the present study, isolated NDRD (Group 1) was found in $51.2 \%$ (20) of the patients. In patients with isolated NDRD, primary glomerular diseases were most common. Among them 45\% (9) had primary glomerular diseases (PGD), 30\% had tubulointerstitial diseases (TID), 15\% (3) had secondary glomerular diseases (SGD), 10\% (2) had vascular disease. IgA Nephropathy was the most common PGD affecting $33.3 \%$ (3) of patients, followed by Focal Segmental Glomerulosclerosis (FSGS) in 22.2\%(2), Infection related Glomerulonephritis (IRGN) in $11.1 \%(1)$, Minimal Change
Disease (MCD) in 11.1\%(1), Membranous nephropathy $(\mathrm{MN})$ in $11.1 \% \quad(1)$, Membrano proliferative Glomerulonephritis (MPGN) in $11.1 \%$ (1) of patients.

In present study, primary glomerular diseases were more common in isolated NDRD group. Among them, IgA Nephropathy was the most common glomerular disease in our study. According to previous reports on NDRD in patients with type $2 \mathrm{DM}$, patients in south east Asia, namely South Korea, China, Hong Kong had high incidence of $\operatorname{IgA}$ Nephropathy ${ }^{[20]}$. In contrast, a recent study from United states, by Pham et al., reported FSGS as the most common NDRD ${ }^{[10]}$. The differences in prevalence patterns of glomerulopathies among various populations in patients with type 2 DM may reflect hereditary and racial predispositions for specific glomerulopathies.

In group 1, ATIN was the most common TID found in $66.6 \%$ (4) of patients, followed by ATN in $16.6 \%(1)$, Cast nephropathy in $16.6 \%(1)$ of patients. The secondary glomerular diseases are lupus nephritis affecting 33.3\% (1), antineutrophil cytoplasmic antibody (ANCA) related pauciimmune glomerulonephritis in $33.3 \%$ (1), Thrombotic microangiopathy (TMA) in $33.3 \%$ (1) of patients. In vascular disease, benign nephrosclerosis was present in $20 \%$ (2) of patients.

In the present study, NDRD with underlying DN (Group 2) was found in $20.5 \%$ (8) of the patients. In patients with NDRD with underlying DN, tubulointerstitial diseases were most common. Among them 62.5\% (5) had tubulointerstitial diseases (TID), 37.5\% (3) had primary glomerular diseases (PGD). Acute tubulointerstitial nephritis (ATIN) was the most common TID found in 60\%(3) of patients, followed by acute tubular necrosis (ATN) in 40\%(2) patients. IRGN was the most common PGD found in $66.6 \%$ (2), followed by IgA Nephropathy in $33.3 \%$ (1).

Our study was in accordance with Soni et al. ${ }^{[7]}$ from south India, in their study observed that the most common NDRD were acute interstitial nephritis $(18.1 \%)$, followed by postinfectious glomerulonephritis (17.24\%), membranous nephropathy (11.20\%) and focal segmental glomerulosclerosis (7.75\%). Glomerulonephritis as the most common change ${ }^{[12]}$.

Table 13: Comparison of common NDRD lesion in various studies

\begin{tabular}{|c|c|c|c|c|}
\hline & Present study & Soni $\boldsymbol{e t}$ al. ${ }^{[7]}$ & ${\text { Prakash } \text { et al. }{ }^{[6]}}$ Sharma SG et al. ${ }^{[19]}$ \\
\hline Common lesion & Acute tubulointerstitial nephritis & Acute interstitial nephritis & Tubulointerstitial nephritis & Acute Tubular Necrosis \\
\hline
\end{tabular}

In contrary to these, Hashim et al. ${ }^{[32]}$ from Iraq reported MPGN (40\%) as the commonest followed by focal segmental glomerulosclerosis (FSGS) (20\%), MN (25\%), MCD (10\%), and amyloidosis (5\%). In a study from Italy where Mazzucco et al. ${ }^{[33]}$ analyzed kidney biopsies of 393 patients of type $2 \mathrm{DM}$ observed $\mathrm{MN}(28.4 \%)$ as the most common PGD followed by IgA Nephropathy (22\%), MCD/FSGS (20\%), and PIGN (10.1\%).

Studies from Korea and China reported IgA Nephropathy as the commonest NDRD accounted for $59 \%$ patients ${ }^{[8,9]}$. A recent Chinese study also reported similar result ${ }^{[20]}$. A study from Thailand showed MN as commonest NDRD, while in Kuwait, it was Crescentic Glomerulonephritis [34]. These results suggest that prevalence of different biopsy proven renal diseases in diabetic patients depends on the usual prevalence of renal disease in the total population according to the geographical area and ethnic characteristics and NDRD is merely a coincidental in type 2 DM.

A number of modified proteins, like oxidized low density lipoproteins that develop in diabetes are potentially immunogenic resulting in immune complex generation and inflammation. Circulating immune complexes and glomerular IgG deposits especially the proinflammatory subtypes IgG1 and IgG3 isotypes are recognized in diabetic experimental models. Enhanced exposure of antigenic cellular components and preexisting glomerular alterations might favor an immune reaction in the subepithelial space ${ }^{[1,}$ 7].

A renal biopsy is rarely performed to confirm the etiology because there is no formal justification or uniform pathological classification system for DN that is closely related to clinical renal outcomes or that may improve clinical management ${ }^{[16]}$. However, recent several studies of 
renal biopsies from patients with type $2 \mathrm{DM}$ and renal disease have revealed a heterogeneous group of disease entities ${ }^{[7,20]}$. Furthermore, because renal survival can be prolonged with early initiation of disease-specific therapy in patients with DM and NDRD, an accurate diagnosis is crucial, necessitating established indications for renal biopsy in patients with type $2 \mathrm{DM}$ and renal involvement. In our study, short duration of diabetes, absence of retinopathy and active urine sediment are factors associated with NDRD. Hence, for patients having these factors, a renal biopsy should be recommended to allow for precise diagnosis and prompt treatment, leading to better renal survival and the avoidance of ESRD.

\section{Limitations of the study}

1. Small sample size

2. Lack of electron microscopic study of kidney tissue

3. Biopsy based clinicopathologic studies suffer from selection bias. So our data can only be applied to those patients who have high pre-test probability of NDRD

\section{Conclusion}

This study demonstrates that the prevalence of heterogenous non diabetic renal diseases are more compared to traditional diabetic nephropathy in patients with type 2 DM with atypical clinical renal disease. To conclude, it is difficult to differentiate NDRD from DN merely on the basis of clinical and laboratory criteria and kidney biopsy is an important diagnostic tool to define underlying disease and to identify the treatable causes.

\section{Acknowledgment}

The author is thankful to Department of Nephrology for providing all the facilities to carry out this work.

\section{Conflict of Interest: None \\ Funding Support: Nil}

\section{References}

1. Ghani AA, Al Waheeb S, Al Sahow A, Hussain N. Renal biopsy in patients withtype 2 DM: Indications and nature of the lesions. Ann Saudi Med 2009;29:4503.

2. Beck MJ, Evans BJ, Quarry- Horn JL, Kerrigan JR. Type 2 Diabetes Mellitus: Issues for the medical care of pediatric and adult patients. South Med J 2002;95:9921000.

3. Martinez-Castelao A, Navarro-Gonzalez JF, Gorriz JL et al. The concept and the epidemiology of diabetic nephropathy have changed in recent years. J Clin Med 2015;4:1207-1216.

4. Deger SM, Ellis CD, Bian A et al. Obesity, diabetes and survival in maintenance hemodialysis patients. Ren Fail 2014;36:546-551

5. Vijayan $\mathrm{M}$, Radhakrishnan S, Abraham $\mathrm{G}$ et al. Diabetic kidney disease patients on hemodialysis: a retrospective survival analysis across different socioeconomic groups. Clin Kidney J 2016;9:833-838

6. Prakash J, Lodha M, Singh SK, Vohra R, Raja R, Usha. Diabetic retinopathy is a poor predictor of type of nephropathy in proteinuric type 2 diabetic patients. J Assoc Physicians India 2007;55:412- 6.
7. Soni SS, Gowrishankar S, Kishan AG, Raman A. Non diabetic renal disease in type 2 diabetes mellitus. Nephrology (Carlton) 2006;11:533- 7.

8. Mak SK, Gwi E, Chan KW, Wong PN, Lo KY, Lee KF et al. Clinical predictors of non- diabetic renal disease in patients with non-insulin dependent diabetes mellitus. Nephrol Dial Transplant 1997;12:2588- 91.

9. Lee EY, Chung $\mathrm{CH}$, Choi SO. Non- diabetic renal disease in patients with non- insulin dependent diabetes mellitus. Yonsei Med J 1999;40:321 - 6.

10. Pham TT, Sim JJ, Kujubu DA, Liu IL, Kumar VA. Prevalence of nondiabeticrenal disease in diabetic patients. Am J Nephrol 2007;27:322- 8.

11. Olson JL, Laszik ZG. Diabetic Nephropathy. Heptinstall's Pathology of the Kidney. Jennette JC, Heptinstall RH, editors, $6^{\text {th }}$ ed. Philadelphia: Lippincott Williams \& Wilkins 2007, 801- 52.

12. Prakash J, Sen D, Usha, Kumar NS. Non-diabetic renal disease in patients with type 2 diabetes mellitus. $\mathrm{J}$ Assoc Physicians India 2001;49:415- 20.

13. Olsen S, Mogensen CE. How often is NIDDM complicated with non- diabetic renal disease? An analysis of renal biopsies and the literature. Diabetologia 1996;39:1638-45.

14. Wilfred DC, Mysorekar VV, Venkataraman RS, Eshwarappa M, Subramanyan R. Nondiabetic renal disease in type 2 diabetesmellitus patients: A clinicopathological study. J Lab Physicians 2013;5:94- 9 .

15. Tone A, Shikata K, Matsuda M, Usui H, Okada S, Ogawa D et al. Clinical features of non- diabetic renal diseases in patients withtype 2 diabetes. Diabetes Res Clin Pract 2005;69:237- 42.

16. Chang TI, Park JT, Kim JK, Kim SJ, Oh HJ, Yoo DE et al. Renal outcomes in patients with type 2 diabetes with or without coexisting non-diabetic renal disease. Diabetes Res Clin Pract 2011;92:198-204.

17. Wong TY, Choi PC, Szeto CC, To KF, Tang NL, Chan AW et al. Renal outcome in type 2 diabetic patients with or without coexisting non-diabetic nephropathies. Diabetes Care 2002;25:900-5.

18. Gambara V, Mecca G, Remuzzi G, Bertani T. Heterogeneous nature of renal lesions in type II diabetes. J Am SocNephrol 1993;1458-1466.

19. Sharma SG, Bomback AS, Radhakrishnan J et al. The modern spectrum ofrenal biopsy findings in patients with diabetes. Clin J Am SocNephrol 2013;8:17181724.

20. Zhou J, Xiangmei C, Xie Y et al. A differential diagnostic model of diabetic and non-diabetic renal diseases. Nephrol Dial Transplant 2008;23:1940-45.

21. Das U, Dakshinamurty KV, Prayaga A, Uppin MS. Nondiabetic kidney disease in type 2 diabetic patients: A single center experience. Indian $\mathbf{J}$ Nephrol 2012;22:358- 62 .

22. Unnikrishnan RI, Rema M, Pradeep R, Deepa M, Shanthirani CS, Deepa $\mathrm{R}$ et al. Prevalence and risk factor of diabetic nephropathy in an urban south Indian population; The Chennai Urban Rural Epidemiology study (CURES - 45). Diabetes Care 2007;30:2019- 24.

23. KittraweeKritmetapak, Sirirat Anutrakulchai, Chatlert Pongchaiyakul et al. Clinical and pathological characteristics of non-diabetic renal disease in type 2 diabetes patients; CKJ 2018, 344-5. 
24. Li H, Li XW, Huang QY, Ye WL, Duan L, Li Y. Non- diabetic renal disease in type II diabetes mellitus. Zhongguo Yi XueKeXue Yuan Xue Bao 2003;25:101- 4 .

25. Castellano I, Covarsí A, Novillo R, Gomez- Martino JR, Ferrando L. Renal histological lesions in patients with type II diabetes mellitus. Nefrologia 2002;22:162- 9 .

26. Lin YL, Peng SJ, Ferng SH, Tzen CY, Yang CS. Clinical indicators which necessitate renal biopsy in type 2 diabetes mellitus patients with renal disease. Int $\mathbf{J}$ Clin Pract 2009;63:1167- 76.

27. Lai CP, Chang CC, Yang Y, Wen Y K, Chiou PF, Chen $\mathrm{M}$ et al. Non- diabetic renal diseases in type 2 diabetic patients with renal involvement: Clinicopathological study in a single medical center in Taiwan. ActaNephrologica 2010;24:157- 66.

28. Okada T, Nagao $\mathrm{T}$, Matsumoto $\mathrm{H}$ et al. Clinical significance of microscopic haematuria in diabetic nephropathy in type 2 diabetes patients with overt proteinuria. Nephrology (Carlton) 2013;18:563-568.

29. Matsumura N, Hanatani M, Nishino $\mathrm{T}$ et al. [The clinicopathological significance of hematuria in diabetics]. Nihon Jinzo Gakkai Shi 1994;36:1036-1045.

30. Gall MA, Rossing P, Skøtt P, Damsbo P, Vaag A, Bech $\mathrm{K}$ et al. Prevalence of micro andmacroalbuminuria, arterial hypertension, retinopathy and large vessel disease in European type 2 (non- insulin- dependent) diabetic patients. Diabetologia 1991;34:655- 61.

31. Parving HH, Gall MA, Skøtt P, Jørgensen HE, Løkkegaard H, Jørgensen F et al. Prevalence and causes of albuminuria in non- insulin-dependent diabetic patients. Kidney Int 1992;41:758- 62.

32. Ali H, Anwar M, Ahmad T, Chand N. Diabetes Mellitus from antiquity to present scenario and contribution of Greco- Arab physicians. JISHIM 2006;5:46-50.

33. Mazzucco G, Bertani T, Fortunato M, Bernardi M, Leutner M, Boldorini R et al. Different patterns of renal damage in type 2 diabetes mellitus: A multicentric study on 393 biopsies. Am J Kidney dis 2002;39:713-20.

34. Chawarnkul O, Vareesangthip K, Ongajyooth L, Cheunsuchon B, Parichatikanond P. Non-diabetic glomerular disease in Type II DM: 10 years experience. J Med Assoc Thai 2009;92:S57- 60. 